\title{
Proteomic analysis of urine reveals biomarkers for the diagnosis and phenotyping of abdominal-type Henoch-Schonlein purpura
}

\author{
Lulu Jia ${ }^{1 \#}$, Jianqiang Wu ${ }^{2 \#}$, Jing Wei ${ }^{3 \#}$, Lina Du ${ }^{4 \#}$, Panpan Wang ${ }^{4}$, Yanju Zhang ${ }^{1}$, Yuncui Yu ${ }^{1}$, \\ Xiaoling Wang ${ }^{1}$, Yan Yang ${ }^{4}$, Youhe Gao ${ }^{3}$
}

${ }^{1}$ Clinical Research Center, National Center for Children's Health, Beijing Children's Hospital, Capital Medical University, Beijing, China; ${ }^{2}$ Medical Research Center, Peking Union Medical College Hospital, Chinese Academy of Medical Sciences \& Peking Union Medical College, Beijing, China; ${ }^{3}$ Department of Biochemistry and Molecular Biology, School of Life Sciences, Beijing Normal University, Gene Engineering Drug and Biotechnology Beijing Key Laboratory, Beijing, China; ${ }^{4}$ Department of Chinese Medicine, National Center for Children's Health, Beijing Children's Hospital, Capital Medical University, Beijing, China

Contributions: (I) Conception and design: L Jia, Y Yang, Y Gao; (II) Administrative support: X Wang; (III) Provision of study materials or patients: L Du, P Wang, Y Zhang, Y Yu; (IV) Collection and assembly of data: J Wu, J Wei; (V) Data analysis and interpretation: L Jia, J Wu, J Wei; (VI) Manuscript writing: All authors; (VII) Final approval of manuscript: All authors.

"These authors contributed equally to this work.

Correspondence to: Professor Yan Yang. No. 56 Nanlishi Street, Beijing 100045, China. Email: yy2303@sina.com; Professor Youhe Gao. No. 19 Xinjiekouwai Street, Beijing 100875, China. Email: gaoyouhe@bnu.edu.cn.

Background: Abdominal-type Henoch-Schonlein purpura (HSP) is a common refractory disease in children. Currently, no specific diagnostic biomarker is available for HSP.

Methods: Children with abdominal type HSP were first diagnosed with three syndromes using Chinese traditional medicine. The urinary proteomes among the three syndromes of patients with abdominal type HSP and healthy controls were compared using two label-free proteomics quantifications, including datadependent acquisition and data-independent acquisition.

Results: For the comparison between patients with abdominal type HSP and healthy children, a total of 75 differential urinary proteins were identified by determining the overlap of the two experiments. The ingenuity pathway analysis (IPA) analysis showed that these differential proteins were correlated with the pathogenesis of abdominal type HSP. Of these, 37 proteins were distributed in 13 solid tissues as tissue-enriched proteins. Monitoring changes in these proteins might help us detect uncommon clinical manifestations of HSP. Patients with abdominal type HSP can be further distinguished into three syndromes based on the urine proteome. Finally, a panel of six urinary proteins (P25774, P09417, Q7Z5L0, P60900, P14550 and P09668) was constructed for both the diagnosis and phenotyping of abdominal type HSP.

Conclusions: Urinary protein biomarkers for the diagnosis and phenotyping of abdominal type HSP were identified, which will contribute to the personalized treatment of patients with abdominal type HSP.

Keywords: Henoch-Schonlein purpura (HSP); urine; proteomics; biomarkers; precision treatment

Submitted Oct 19, 2020. Accepted for publication Jan 17, 2021.

doi: $10.21037 / \mathrm{tp}-20-317$

View this article at: http://dx.doi.org/10.21037/tp-20-317

\section{Introduction}

Henoch-Schonlein purpura (HSP) is the most common systemic vasculitis in children, with an incidence of 10-20 cases per 100,000 per year (1). The dominant clinical features include cutaneous purpura, arthritis, abdominal pain, gastrointestinal bleeding, and nephritis (2). Threequarters of children with HSP develop abdominal symptoms, including diarrhea, abdominal pain, vomiting, gastrointestinal bleeding and hematochezia, and even intussusception and intestinal perforation $(3,4)$. These patients can be classified as 
abdominal-type HSP. Abdominal type HSP is an acute and severe disease in pediatric patients, and severe cases can be secondary to intestinal obstruction and intussusception.

Currently, there are no specific diagnostic laboratory abnormalities for HSP. The serum IgA concentration is increased in $50 \%$ of patients, and serum $\mathrm{C} 3$ or $\mathrm{C} 4$ concentrations is low in a few patients. Other laboratory studies are useful only to exclude other conditions that may resemble HSP (5-8). The diagnosis of HSP is mainly based on the clinical manifestation. However, the abdominal symptoms often precede purpura in children with abdominal type HSP, and the clinical manifestation lacks specificity, which means that abdominal type HSP cannot be diagnosed early and is easily misdiagnosed. Diagnostic biomarkers for abdominal type HSP urgently need to be developed.

Information about the effective treatment for HSP is limited. Anti-infective, anti-allergic and symptomatic treatment are now dominant. Corticosteroid therapy has recently emerged as an effective treatment for abdominal pain (9). However, there is no evidence that corticosteroid therapy is effective in treating purpura, shortening the duration of the disease, or preventing recurrence (5). In addition, long-term use will lead to more serious adverse reactions. To reduce the incidence of adverse reactions and improve efficacy, corticosteroid therapy is often combined with Chinese herbs to treat abdominal type HSP in China.

Several studies have shown that corticosteroid therapy combined with Chinese herbs is more effective and safer than corticosteroid therapy alone $(10,11)$. However, it is interesting that corticosteroid therapy is not combined with the same Chinese herbs for all patients with abdominal type HSP. In China, patients with abdominal type HSP will be divided into different syndromes according their clinical symptoms, such as the location and appearance of purpura, fever, cough, changes in stool characteristics, changes in tongue coating and pulse manifestations. Based on these different manifestations, three subtypes of HSP can be distinguished by expert doctors and then patients will be received significantly different drug prescriptions.

This inspired us to further divide abdominal type HSP into different types based on molecular phenotype. To achieve this aim, we adopted systems biology research methods. Urine is one of the main excreted human body fluids. Due to the lack of homeostatic mechanisms, it exhibits substantial changes in the body. Therefore, we hypothesized that the differences among different syndromes of patients with abdominal type HSP would be reflected in the urine.
Proteins are one of the main components of urine. The urinary proteome is mainly composed of plasma proteins that pass through the glomerular barrier and proteins shed by cells within the urogenital system (12). It can reflect the changes in both systemic and local conditions of the body. With the development of MS techniques, more than 6,000 proteins have been identified in the normal human urinary proteome (13). Therefore, biomarker discovery through urine proteomics has become a very popular field and is also of great significance for early diagnosis, for clarifying the classification problem, and for better understanding the pathogenesis of abdominal type HSP.

In this study, urine samples were collected from children with abdominal type HSP with different syndromes under the guidance of traditional Chinese medicine. The urine proteomes were compared with the healthy controls by quantitative proteomics techniques to investigate whether patients with abdominal type HSP or the syndromes can be distinguished based on the urine proteome profile. Some differential urinary proteins with the potential for diagnosis and phenotyping of abdominal type HSP were identified, which might help guide drug therapy for abdominal type HSP in children.

We present the following article in accordance with the MDAR reporting checklist (available at http://dx.doi. org/10.21037/tp-20-317).

\section{Methods}

\section{Participants and sample collection}

Patients with abdominal type HSP and healthy controls were recruited from Beijing Children's Hospital. These children were all aged from 1 to 10 years. Abdominal type HSP was diagnosed using the international criteria for HSP with abdominal symptoms. Children with abdominal type HSP were further diagnosed with three syndromes [wind-heat (WH) syndrome, damp-poison (DP) syndrome, and spleendeficiency (SD) syndrome] based on traditional Chinese medicine by experienced Chinese medicine doctors (14). Patients with hematuria, severe proteinuria, and other diseases were excluded. Urine samples were collected from patients with abdominal type HSP and healthy controls. This study was conducted in accordance with the Declaration of Helsinki (as revised in 2013). The study was approved by the local ethics committee of Beijing Children's Hospital of Capital Medical University (No. 2016-91) and informed consent was taken from all the patients. 


\section{Classification standard of abdominal type HSP syndromes based on traditional Chinese medicine}

\section{WH syndrome}

Patients have fever, slight chills, cough, and loss of appetite. Purpura occurs in the lower body, especially the lower limbs and buttocks. They are often symmetrical, bright red, present as papules or erythema, and vary in size and shape. They can be fused into a patch or itchy. Facial swelling, abdominal pain and bloody stool can also be observed. A red tongue with a thin greasy coating could be observed, and a superficial and rapid pulse may be felt (14).

\section{DP syndrome}

Skin of the lower extremities is full of purple freckle spots. Some symptoms can be observed, such as abdominal pain, bad breath, poor appetite, gastric fullness, bleeding gums, loose stool that is dark red or brown-purple or with roundworms. A red tongue with yellow coating can be observed, and a slippery pulse may be felt (14).

\section{SD syndrome}

The purpura is often pale red or recurrent. Patients have some symptoms, such as weight loss, sallow complexion, lassitude, general weakness, poor appetite, spontaneous sweating, low urine output, loose stool, abdominal pain, or even edema of the whole body or lower extremities. A pale tongue with a thin white coating could be observed, and a thready weak pulse or deep weak pulse may be felt (14).

\section{Urine sample preparation}

Midstream first morning urine was collected from these subjects with no serial measurements. All the urine samples of patients were collected when they first visit the hospital before treatment. After collection, the urine was immediately centrifuged at $5,000 \mathrm{~g}$ for $30 \mathrm{~min}$ at $4{ }^{\circ} \mathrm{C}$ to remove the cell debris. Protein from individual urine samples $(30 \mathrm{~mL})$ was precipitated with ethanol $(90 \mathrm{~mL})$ overnight at $-20{ }^{\circ} \mathrm{C}$ followed by centrifugation at $10,000 \mathrm{~g}$ for $30 \mathrm{~min}$ to remove the supernatant. The precipitate was then resuspended in lysis buffer $(8 \mathrm{M}$ urea, $2 \mathrm{M}$ thiourea, $50 \mathrm{mM}$ Tris and $25 \mathrm{mM}$ DTT). The protein amount of each sample was measured using the Bradford method. Then, 100 $\mu \mathrm{g}$ of protein from each individual sample was prepared using the filter-aided sample preparation method (FASP) for tryptic digestion (15). The peptide concentration was measured using the BCA method. Finally, $1 \mu \mathrm{g}$ of each peptide sample was used for the subsequent liquid chromatography-tandem mass spectrometry (LC-MS/MS) analysis.

\section{$L C-M S / M S$}

Digested peptides were dissolved in $0.1 \%$ formic acid and loaded onto a trap column $(75 \mu \mathrm{m} \times 2 \mathrm{~cm}, 3 \mu \mathrm{m}, \mathrm{C} 18,100 \AA$ ) . The elute was separated on a reverse-phase $\mathrm{C} 18$ analytical column $(50 \mu \mathrm{m} \times 250 \mathrm{~mm}, 2 \mu \mathrm{m}, \mathrm{C} 18,100 \AA)$ using the EASY-nLC 1200 HPLC system (Thermo Fisher Scientific, Waltham, MA, USA). Then, the peptides were analyzed with an Orbitrap Fusion Lumos Tribrid mass spectrometer (Thermo Fisher Scientific, Waltham, MA, USA).

In the data-dependent acquisition (DDA) experiment, the elution was set to $5-30 \%$ buffer B $(0.1 \%$ formic acid in $99.9 \%$ acetonitrile; flow rate of $0.3 \mu \mathrm{L} / \mathrm{min}$ ) for $90 \mathrm{~min}$. The MS parameters were set as follows: the full MS scan was acquired from 150 to $2,000 \mathrm{~m} / \mathrm{z}$ with a resolution of 120,000 , the MS/MS scan was acquired from 110 to 2,000 $\mathrm{m} / \mathrm{z}$ with a resolution of 30,000 in Orbitrap, MS data were acquired in high-sensitivity mode, and 30\% higher-energy collision dissociation (HCD) collision energy and chargestate screening $(+2$ to +7$)$ were used.

In the data-independent acquisition (DIA) experiment, the LC settings were the same as in the DDA experiment to maintain retention time stability. The calibration kit (iRT kit from Biognosys, Switzerland) reagent was spiked at a concentration of 1:20 v/v in all samples. The iRT standard peptides were spiked into the urinary peptides for spectral library generation, and the following single DIA runs were used to calibrate the retention time of extracted peptide peaks. First, to generate the spectral library, all 39 samples were pooled as a mixture and fractionated by a high-pH reversed-phase peptide fractionation kit with 10 fractions. These ten fractions from the spin column were analyzed with a mass spectrometer in DDA mode. Then, the mass spectrometer was changed to the DIA mode. A variable isolation window DIA method with 45 windows was developed. The full scan was set at a resolution of 60,000 over a $\mathrm{m} / \mathrm{z}$ range of 350 to 1,400 , followed by DIA scans with a resolution of 30,000 . The HCD collision energy was set to $30 \%$. The maximal injection time was $50 \mathrm{~ms}$ for the full scan and DIA scans. The precursor ion number was equalized in each isolation window according to the precursor $\mathrm{m} / \mathrm{z}$ distribution of the pooled sample. During the DIA analysis, a pooled sample was inserted as the quality control (QC). 


\section{Data processing}

For the DDA experiment, the raw MS files were searched against the Swiss-Prot Human database using Mascot software (version 2.6.1, Matrix Science, London, UK). The parent ion tolerance was set at $10 \mathrm{ppm}$, and the fragment ion mass tolerance was set to $0.05 \mathrm{Da}$. A maximum of two missed cleavage sites in the trypsin digestion was allowed. Carbamidomethylation of cysteines was set as a fixed modification, and the oxidation of methionine was considered a variable modification. Labelfree quantitation of the proteomic data was performed using Scaffold software (version 4.8.4, Proteome Software Inc., Portland, OR, USA). Peptide identifications were accepted with $\geq 2$ unique peptides at a $1.0 \%$ false discovery rate (FDR) using the Scaffold Local FDR algorithm. Protein identifications were also accepted at an FDR of less than $1.0 \%$. Spectral counts in different samples were compared after normalization with the total spectra according to previously described procedures (16).

For the DIA experiment, first, for library generation, the raw DDA MS files from the ten fractions were processed using Proteome Discoverer (Version 2.1, Thermo, USA) software with SEQUEST HT against the SwissProt database (Homo sapiens, year 201803) appended with the iRT fusion protein sequence. The search allowed two missed cleavage sites in trypsin digestion. Carbamidomethyl (C) was specified as the fixed modification. Oxidation (M) was specified as the variable modification. The parent ion mass tolerances were set to $10 \mathrm{ppm}$, and fragment ion mass tolerance was set to $0.02 \mathrm{Da}$. The $\mathrm{Q}$ value (FDR) cutoff at the precursor and protein levels was $1 \%$. Finally, a total of 3,350 protein groups, 19,684 peptide groups and 61,401 peptide-spectrum matches (PSMs) were identified. Then, the results were imported to Spectronaut Pulsar X (Biognosys AG, Switzerland) software to generate the spectra library (17).

Then, all the raw DIA files were imported into Spectronaut Pulsar X as default settings. The optimal XIC extraction window was determined according to the iRT calibration strategy. The mass tolerance strategy was set to dynamic based on the extensive mass calibration. The cross-run normalization was set to the local normalization based on the local regression (18) Protein inference was performed using the implemented IDPicker algorithm (19). The $\mathrm{Q}$ value was set to 0.01 for data filtering. The sum peak areas of the respective fragment ions in MS2 were used for the quantification of peptide intensities. For later analysis, we required the proteins to be removed if the coefficient of variation $(\mathrm{CV})$ in mixed samples were more than $30 \%$ or the missing values were present in more than 12 samples.
Then, the missing values of protein abundance were filled with the K-nearest neighbors (KNN) method.

\section{Statistical analysis}

Average normalized spectral counts were used for the following DDA statistical analysis, and the normalized peak areas were used for DIA statistical analysis. Data were analyzed using SPSS statistical software (version 22.0). For comparisons between two groups, unpaired $t$-tests were used. For comparisons between more than two groups, analysis of variance (ANOVA) followed by a least significant difference (LSD) post-hoc test was used. Differences were considered significant when $\mathrm{P}<0.05$.

To identify potential urine proteins to differentiate patients with abdominal type HSP from healthy controls, differential proteins in both DDA and DIA experiments were identified according to the following criteria: fold change $\geq 1.5$ or $\leq 0.67$. The above two criteria must be met at the same time. To identify potential urine proteins to differentiate the three disease syndromes, differential urinary proteins in patients with abdominal type HSP were further selected based on the following criteria: (I) ANOVA $\mathrm{P}<0.05$ for the three disease syndromes; and (II) $\mathrm{P}$ value $<0.05$, change ratio $>1.5$ in any of these three disease syndromes when compared to each other.

Then, principal component analysis (PCA) and orthogonal projection to latent structures discriminant analysis (O-PLSDA) were conducted in SIMCA 14.1 (Umetrics, Umea, Sweden) based on the selected biomarkers above. The biomarkers with the best predictive value to discriminate between the patients with abdominal type HSP and controls or between the abdominal type HSP with three syndromes were determined using area under the receiver operating characteristic (ROC) curve (AUC) analysis followed by calculations of sensitivity and specificity.

\section{Biomarker annotation and functional analysis}

The information for the urinary disease biomarkers was acquired from the Urinary Protein Biomarker Database (UPBD) (http://upbd.bmicc.cn/biomarker/web/ indexdb). The information for the tissue specificity of the proteins was acquired from The Tissue Atlas (https:// www.proteinatlas.org/humanproteome/tissue), which contains information regarding the expression profiles of human genes at both the mRNA and protein levels. The classification and criteria are as follows. Tissue enriched: 
Table 1 Clinical characteristics of subjects in this study

\begin{tabular}{|c|c|c|c|c|c|c|c|c|c|c|}
\hline Variable & \multicolumn{5}{|c|}{ DDA method $(n=20)$} & \multicolumn{5}{|c|}{ DIA method $(n=39)$} \\
\hline Number & 5 & 5 & 5 & 5 & - & 9 & 11 & 10 & 9 & - \\
\hline $\operatorname{Sex}(M / F)$ & $2 / 3$ & $3 / 2$ & $4 / 1$ & $3 / 2$ & - & $6 / 3$ & $4 / 7$ & $8 / 2$ & $6 / 3$ & - \\
\hline Age & $7.4 \pm 2.6$ & $8.8 \pm 1.2$ & $7.8 \pm 1.1$ & $6.4 \pm 0.5$ & - & $7.4 \pm 3.2$ & $8.6 \pm 0.7$ & $7.9 \pm 0.7$ & $7.6 \pm 0.8$ & - \\
\hline $\mathrm{N} \%$ & $35-65^{\dagger}$ & $68.2 \pm 3.3$ & $72.9 \pm 6.4$ & $70.2 \pm 3.1$ & 0.577 & $35-65^{\dagger}$ & $71.6 \pm 2.5$ & $70.8 \pm 3.4$ & $69.4 \pm 3.0$ & 0.937 \\
\hline PLT $\left(10^{9} / L\right)$ & $100-400^{\dagger}$ & $392.2 \pm 51.5$ & $467.2 \pm 55.2$ & $403.0 \pm 33.5$ & 0.490 & $100-400^{\dagger}$ & $406.9 \pm 26.7$ & $464.9 \pm 34.5$ & $417.4 \pm 28.5$ & 0.527 \\
\hline $\mathrm{FIB}(g / L)$ & $2-4^{\dagger}$ & $5.5 \pm 1.9$ & $2.8 \pm 0.2$ & $2.6 \pm 0.4$ & 0.079 & $2-4^{\dagger}$ & $4.3 \pm 0.9$ & $3.3 \pm 0.3$ & $3.0 \pm 0.3$ & 0.519 \\
\hline $\mathrm{D}-\mathrm{D}(\mathrm{mg} / \mathrm{L})$ & $0-0.243^{\dagger}$ & $0.92 \pm 0.48$ & $1.66 \pm 0.97$ & $0.59 \pm 0.22$ & 0.976 & $0-0.243^{\dagger}$ & $1.32 \pm 0.36$ & $1.11 \pm 0.36$ & $0.48 \pm 0.12$ & 0.424 \\
\hline
\end{tabular}

Values are given as the mean $\pm \mathrm{SD} .{ }^{\dagger}$, the normal reference values of healthy children; ${ }^{\ddagger}$, comparison of the three syndromes was performed using the Kruskal-Wallis test, followed by post hoc multiple comparisons using the corrected Dunn's test; *, $\mathrm{P}$ value <0.05 between WH and SD. HC, healthy control; WH, wind-heat syndrome; DP, damp-poison syndrome; SD, spleen-deficiency syndrome; WBC, white blood cell; N\%, neutrophilic granulocyte percentage; PLT, blood platelet; FIB, fibrinogen; D-D, D-Dimer; IgA, immunoglobin A.

at least four-fold higher mRNA level in a particular tissue compared to any other tissues. Group enriched: at least four-fold higher average mRNA level in a group of 2-5 tissues compared to any other tissue. Tissue enhanced: at least four-fold higher mRNA level in a particular tissue compared to the average level in all other tissues. Ingenuity pathway analysis (IPA) was used to determine the functional characterization and potential functional interaction for the differential proteins.

\section{Results}

\section{Clinical characteristics of patients with abdominal type HSP and study design}

A total of 20 subjects were included in the DDA experiment, including 5 for each symptom group and 5 for the healthy control group. A total of 39 subjects were included in the DIA experiment, including 11 children with WH syndrome, 10 children with DP syndrome, 9 children with SD syndrome and 9 healthy controls. The clinical characteristics of the patients are shown in Table 1 and table online: https://cdn. amegroups.cn/static/public/tp-20-317-1.pdf. According to Table 1, based on traditional biochemical indicators, the three syndromes of patients with abdominal type HSP could not be distinguished.

In this study, the urinary proteomes among three syndromes of patients with abdominal type HSP and healthy controls were compared. The flow chart of the study design is shown in Figure 1. First, an experiment was initiated in which label-free proteomics quantifications with DDA were used, and 20 urine samples were included (five samples in each group). These subjects were matched by age and sex. Then, a larger sample size experiment was performed that used label-free proteomics quantifications with DIA and included a total of 39 urine samples, that is, 11 patients with $\mathrm{WH}$ syndrome in the $\mathrm{WH}$ group, 10 patients with DP syndrome in the DP group, 9 patients with SD syndrome in the SD group and 9 healthy children in the control group. The consistent comparison results from both experiments were selected for subsequent biomarker discovery analysis.

\section{Identification of the potential diagnostic biomarkers for abdominal type HSP}

To identify the potential diagnostic biomarkers for abdominal type HSP, the urinary proteome of abdominal type HSP children was compared with that of healthy children. In the DDA proteomics quantification experiment, a total of 1,292 urinary protein groups were identified in 20 urine samples. Finally, a total of 174 differential urine proteins were identified between abdominal type HSP and healthy children with a ratio $>1.5$ and $\mathrm{P}$ value $<0.05$ (table online: https://cdn. amegroups.cn/static/public/tp-20-317-2.pdf). Among these differential proteins, 84 urine proteins were upregulated, and 


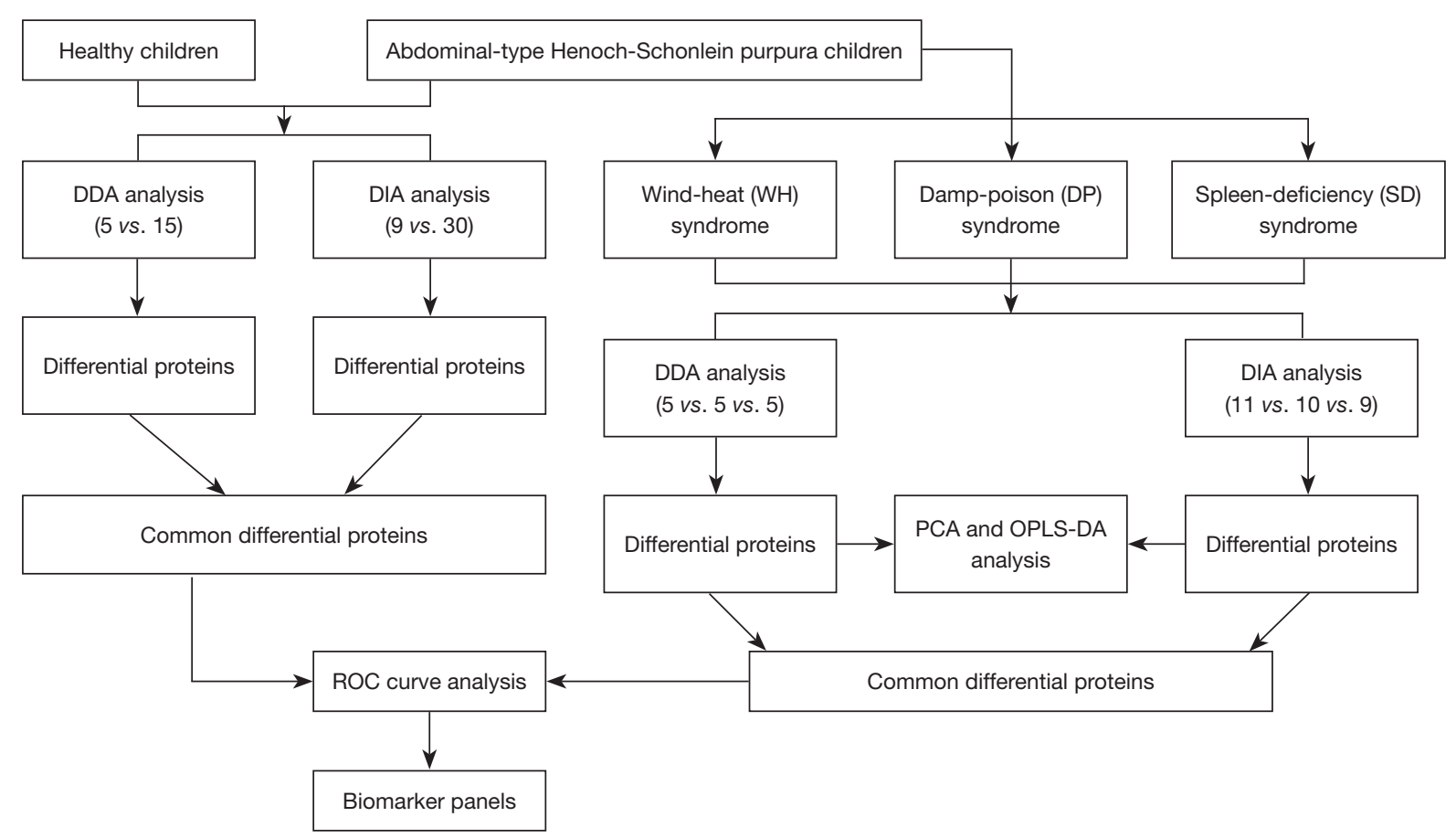

Figure 1 Flow chart of the study design. DDA, data-dependent acquisition; DIA, data-independent acquisition; PCA, principal component analysis; O-PLS-DA, orthogonal projection to latent structures discriminant analysis; ROC, receiver operating characteristic.

90 urine proteins were downregulated.

In the DIA proteomics quantification experiment, a total of 2,366 urinary proteins were identified in 39 urine samples. Specifically, 1,627 proteins were accepted when the $\mathrm{CV}$ values of protein abundance in QC samples and missing values in all samples were both less than $30 \%$. Finally, 340 urinary proteins changed significantly in patients with abdominal type HSP compared with healthy controls (table online: https://cdn.amegroups.cn/static/public/tp20-317-3.pdf). Among these differential proteins, 154 urine proteins were upregulated, and 186 urine proteins were downregulated.

The potential diagnostic biomarkers for abdominal type HSP were identified by comparing the differential proteins identified by the DDA experiment and DIA experiment. Finally, a total of 75 differentially expressed proteins, including 46 commonly upregulated proteins and 29 commonly downregulated proteins, were identified (table online: https://cdn.amegroups.cn/static/public/tp-20-3174.pdf). These proteins have the potential to differentiate patients with abdominal type HSP from healthy children.

\section{Annotation and functional analysis of the potential diagnostic biomarkers for abdominal type HSP}

Whether the potential diagnostic biomarkers of abdominal type HSP found in this study have ever been reported as biomarkers for other diseases was investigated. The UPBD comprehensively collects information on urinary biomarkers or biomarker candidates from published literature. The 75 proteins were searched from the UPBD one by one. Ten proteins have been reported as candidate biomarkers for twenty-one diseases (Table 2).

For speculating target organ damage, the expression levels of protein biomarkers in different tissues are very useful. The Tissue Atlas contains information regarding the expression data of human genes from 44 normal human tissue types at both the mRNA and protein levels. The 75 potential biomarkers were searched from the Tissue Atlas, and their expression-enriched or enhanced tissue types were acquired. A total of 24 tissues were identified (Figure 2A). The biomarker expression of enriched or enhanced tissue may be an organ that abdominal type HSP can affect. We speculated that the more biomarkers there 
Table 2 Ten potential biomarkers for abdominal type HSP were reported as candidate biomarkers for other diseases

\begin{tabular}{|c|c|c|c|}
\hline Protein ID & Disease & Pmid & The potential reason why the abdominal type HSP shared biomarkers \\
\hline P00450 & $\begin{array}{l}\text { Other diseases of intestines } \\
\text { (K55-K64) }\end{array}$ & 21915437 & $\begin{array}{l}\text { Intestinal involvement is the clinical characteristic of the patients included in } \\
\text { this study. The most common complications of abdominal type HSP include } \\
\text { intussusception, perforation, obstruction or gastrointestinal hemorrhage }\end{array}$ \\
\hline P01019 & Henoch-Schonlein purpura nephritis & 21854508 & \multirow{13}{*}{$\begin{array}{l}\text { Henoch-Schonlein purpura, also referred to as IgA vasculitis (IgAV), is } \\
\text { characterized by immunoglobulin A1 (IgA1)-dominant immune deposits (20). } \\
\text { HSP frequently affects kidneys, which is called HSP nephritis. It seems } \\
\text { to share pathological mechanisms with IgA nephropathy (21). The renal } \\
\text { involvement of HSP might share some biomarkers with other kidney } \\
\text { diseases such as acute kidney failure, dent disease, kidney transplant } \\
\text { rejection, and renal cell carcinoma. In addition, several biomarkers of other } \\
\text { disease were shared with biomarkers of IgA glomerulonephritis, which is } \\
\text { associated with pathological progression of HSP }\end{array}$} \\
\hline P01019 & IgA glomerulonephritis & $\begin{array}{l}21366514 \\
25523477\end{array}$ & \\
\hline P04217 & IgA glomerulonephritis & 25957429 & \\
\hline P01019 & Chronic kidney disease & $\begin{array}{l}24065527 \\
24664631\end{array}$ & \\
\hline P04217 & Nephrotic syndrome & 21591266 & \\
\hline P07686 & Proteinuria & 21265931 & \\
\hline P07686 & $\begin{array}{l}\text { Nephropathy induced by other } \\
\text { drugs, medicaments and biological } \\
\text { substances }\end{array}$ & 15967208 & \\
\hline P01019 & Dent disease & 15140760 & \\
\hline P07195 & Dent disease & 15140760 & \\
\hline P07737 & Dent disease & 15140760 & \\
\hline P01011 & Kidney transplant rejection & 17331118 & \\
\hline P39059 & Complications of kidney transplant & 22253069 & \\
\hline P00450 & Renal cell carcinoma & 23511837 & \\
\hline P00450 & Diabetic nephropathy & $\begin{array}{l}17327332 \\
26608305\end{array}$ & \multirow{8}{*}{$\begin{array}{l}\text { HSP is a small vessel vasculitis. Microvasculitis is also one of major } \\
\text { complications of diabetes (24). Vascular damage results in weakening of } \\
\text { walls of the blood vessels and leakage of protein into the tissues, and an } \\
\text { inflammatory response might happen in the pathological process of both } \\
\text { diseases }(24,25) \text {. In addition, many diabetes biomarkers were found to be } \\
\text { shared with biomarkers of kidney diseases, which can also be involved in } \\
\text { the progression of HSP }\end{array}$} \\
\hline P01011 & Diabetic nephropathy & 17327332 & \\
\hline P04217 & Diabetic nephropathy & 17327332 & \\
\hline P00450 & Type 2 diabetes mellitus & 15111541 & \\
\hline P00450 & $\begin{array}{l}\text { Type } 2 \text { diabetes mellitus with diabetic } \\
\text { nephropathy }\end{array}$ & 22536212 & \\
\hline P07686 & Type 2 diabetes mellitus & 15111541 & \\
\hline P01019 & Type 2 diabetes mellitus & 26617876 & \\
\hline P04217 & Type 1 diabetes mellitus & 22678621 & \\
\hline
\end{tabular}

Table 2 (continued) 
Table 2 (continued)

\begin{tabular}{|c|c|c|c|}
\hline Protein ID & Disease & Pmid & The potential reason why the abdominal type HSP shared biomarkers \\
\hline P07737 & Malignant neoplasm of bladder & 22065568 & \multirow{4}{*}{$\begin{array}{l}\text { HSP may affect the bladder, which leads to bladder wall hematomas and } \\
\text { urinary retention (28). In addition, many bladder biomarkers were found to } \\
\text { be shared with biomarkers of kidney diseases, which can also be involved } \\
\text { in the progression of HSP }\end{array}$} \\
\hline P04217 & Malignant neoplasm of bladder & 17518487 & \\
\hline P39059 & Malignant neoplasm of bladder & 23389364 & \\
\hline P07195 & Malignant neoplasm of bladder & 21496341 & \\
\hline
\end{tabular}

were, the greater the possibility.

Then, functional characterization of the differentially expressed proteins was performed using IPA software. The IPA analysis revealed that the differential proteins were linked to three classes of disease and biofunctions, including the immune and inflammatory response class (Figure 2B), lipid and carbohydrate metabolism class (Figure 2C), and organismal injury and abnormalities class (Figure $2 D$ ). In addition, the differentially expressed proteins formed two tightly connected networks, which were associated with cardiovascular disease: organismal injury (Figure $2 E$ ) and abnormal humoral immune response (Figure $2 F$ ).

\section{Investigation of whether abdominal type HSP can be distinguished into different syndromes based on the urine proteome}

In this study, patients with abdominal type HSP were divided into three different syndromes guided by traditional Chinese medicine: WH syndrome, DP syndrome and $\mathrm{SD}$ syndrome). The differential proteins among the three disease syndromes were investigated based on ANOVA.

Differential urinary proteins were selected based on the following criteria: (I) ANOVA $\mathrm{P}<0.05$ for the three disease syndromes; and (II) $\mathrm{P}$ value $<0.05$, change ratio $>1.5$ in any of these three disease syndromes when compared to each other (post-hoc analysis). Finally, 91 and 73 differential proteins were acquired based on DDA and DIA quantification experimental data, respectively (tables online: https://cdn.amegroups.cn/ static/public/tp-20-317-5.pdf). PCA and O-PLS-DA were performed based on differential urinary proteins. The results are shown in Figure 3, indicating that the abdominal type
HSP can be distinguished as three different syndromes based on urinary proteins.

\section{Construction of the urinary protein biomarker panel for the diagnosis and phenotyping of abdominal type HSP}

Only proteins with consistent changes between the DDA experiment and the DIA experiment were selected to determine the phenotyping and diagnosis urinary protein biomarkers. When the abdominal type HSP patients were compared with controls, we found 75 proteins with consistent changes between the two experiments. When the three syndromes were compared, a total of 21 proteins were found to have consistent changes by comparing the results of the DDA experiment and the DIA experiment, including two proteins for the comparison of $\mathrm{WH}$ and DP syndrome, three proteins for the comparison of $\mathrm{WH}$ and SD syndrome, and sixteen proteins for the comparison of DP and SD syndrome (table online: https://cdn.amegroups. cn/static/public/tp-20-317-6.pdf).

To evaluate the diagnostic performance of these candidate biomarkers, which were differentially expressed among the three disease syndromes, ROC curves were plotted. As shown in Figure 4A, a panel of three urinary proteins (P25774 + P09417 + Q7Z5L0) showed excellent sensitivity and specificity for the diagnosis of abdominal type HSP, with an AUC value of 0.966 .

Several urine proteins could be used for distinguishing the three disease syndromes. As shown in Figure 4B, a protein panel (P14550 + P60900) could differentiate WH and DP syndromes with an AUC value of 0.96 . Another protein panel of P25774 and P09668 could differentiate WH and SD 

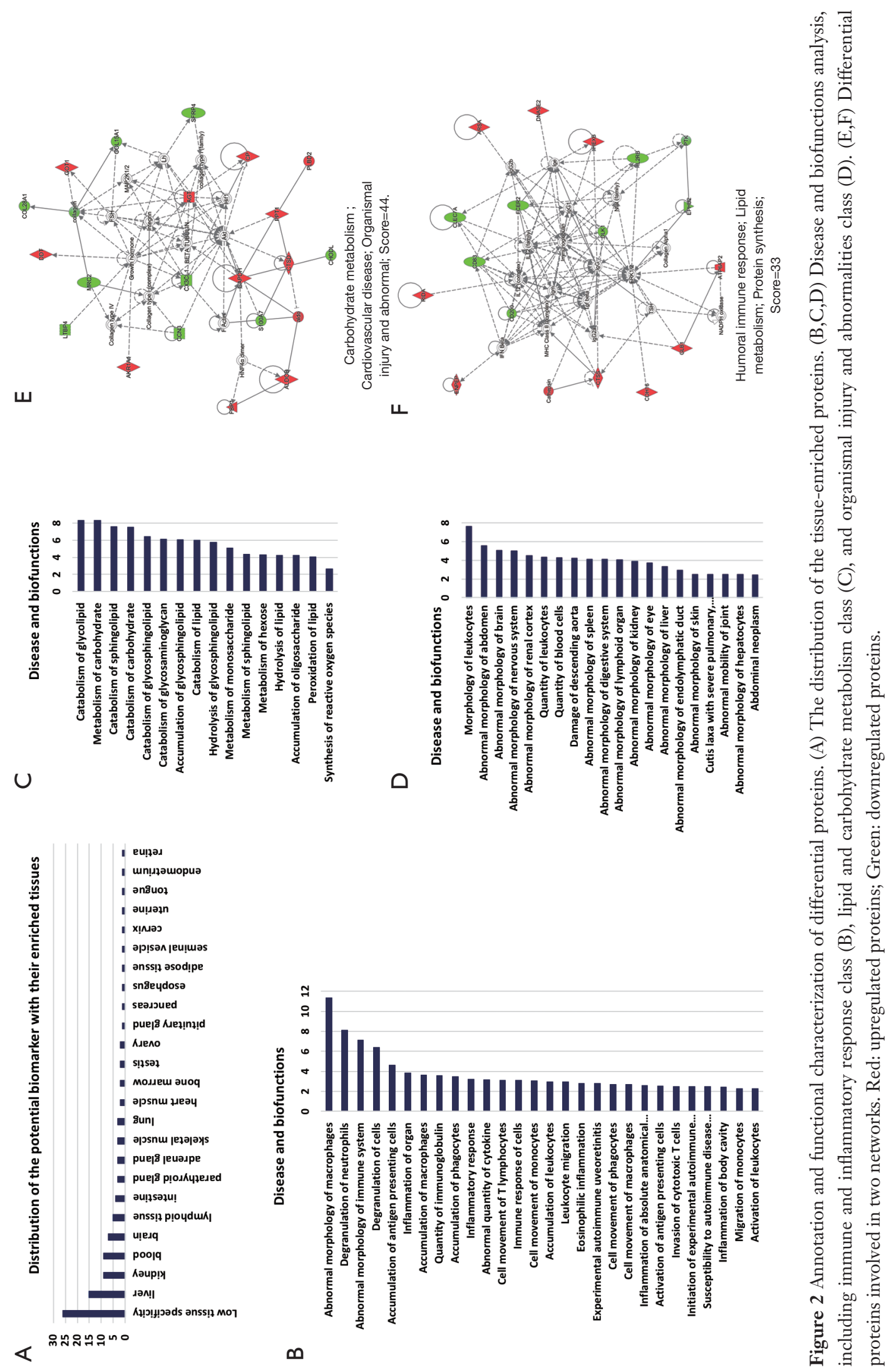
A

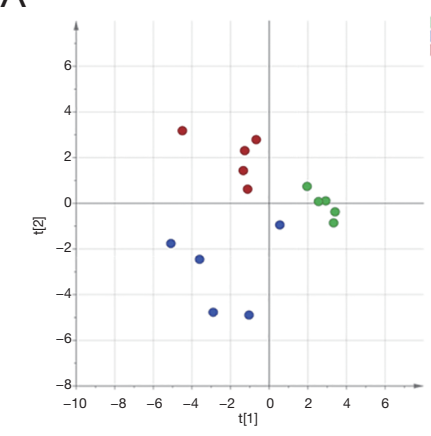

C

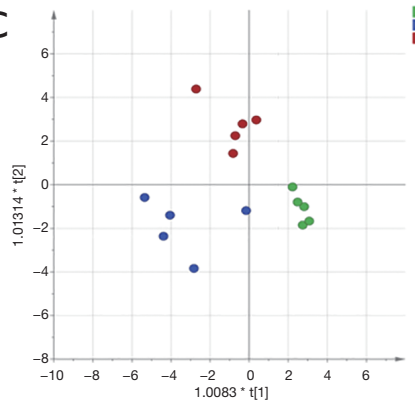

B
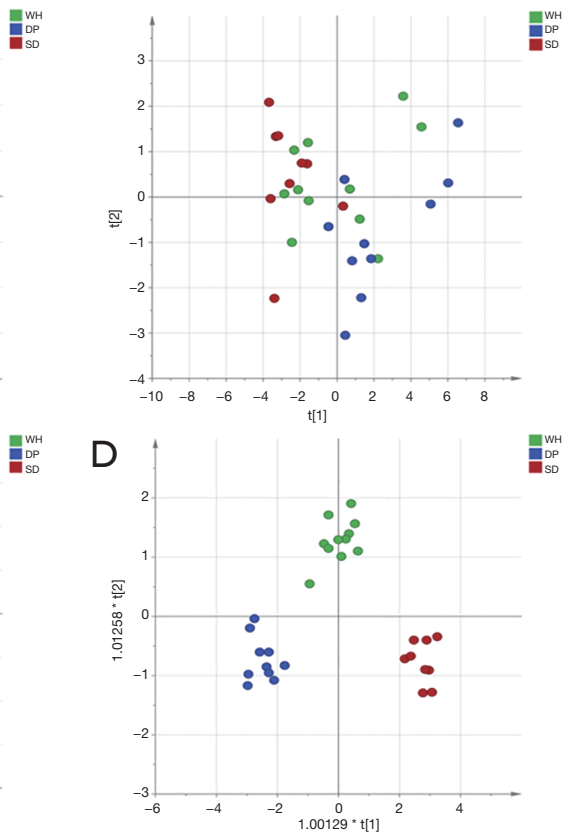

Figure 3 PCA and O-PLS-DA analysis of differential proteins among three disease syndromes of abdominal type HSP. (A) PCA analysis with 91 differential proteins identified by DDA quantification; (B) PCA analysis with 73 differential proteins identified by DIA quantification; (C) O-PLS-DA analysis with 91 differential proteins identified by DDA quantification; (D) O-PLS-DA analysis with 73 differential proteins identified by DIA quantification. Green represents wind-hot syndrome, blue represents damp-poison syndrome, and red represents spleen-deficiency syndrome. WH, wind-heat; DP, damp-poison; SD, spleen-deficiency; PCA, principal component analysis; O-PLS-DA, orthogonal projection to latent structures discriminant analysis; HSP, Henoch-Schonlein purpura; DDA, data-dependent acquisition; DIA, data-independent acquisition.

syndromes with an AUC value of 0.83 (Figure 4C). One urine protein (P09417) could differentiate DP syndrome and SD syndrome with an AUC value of 0.956 (Figure 4D).

These results indicated that a minimum of six urinary proteins (P25774 + P09417 + Q7Z5L0 + P60900 + P14550 $+\mathrm{P} 09668)$ is required to form a urinary protein panel, which has the potential for the diagnosis of abdominal type HSP and the three syndromes of abdominal type HSP.

\section{Discussion}

In the present study, we collected urine samples from healthy children and abdominal type HSP patients with three syndromes according to the guidance of traditional Chinese medicine. The urine proteome was analyzed by two quantitative proteomics experiments. The consistent findings of the two experiments were presented in this study. First, the proteome of the abdominal type HSP patients was compared with that of the controls. By determining the overlap of the different proteins in the two experiments, 75 disease-related proteins were identified. The annotation and function of these 75 proteins were analyzed.

Abdominal type HSP is Henoch-Schonlein purpura, which involves the gastrointestinal tract. HSP is also referred to as IgA vasculitis (IgAV). It is characterized by immunoglobulin A1 (IgA1)-dominant immune deposits affecting small vessels and often involves the skin, gastrointestinal tract, joints, and kidneys (20). Due to the involvement of small blood vessels throughout the body, this disease can cause a variety of symptoms in different organs.

Ten of 75 proteins have been reported as candidate biomarkers for twenty-one diseases. Most proteins have been reported as candidate biomarkers for more than one disease. This suggests that similar pathological damage may exist among these diseases, and the differential proteins can reflect the changes caused by such pathological damage. In this case, we could not determine which disease was the primary cause of the pathological damage. However, 
A

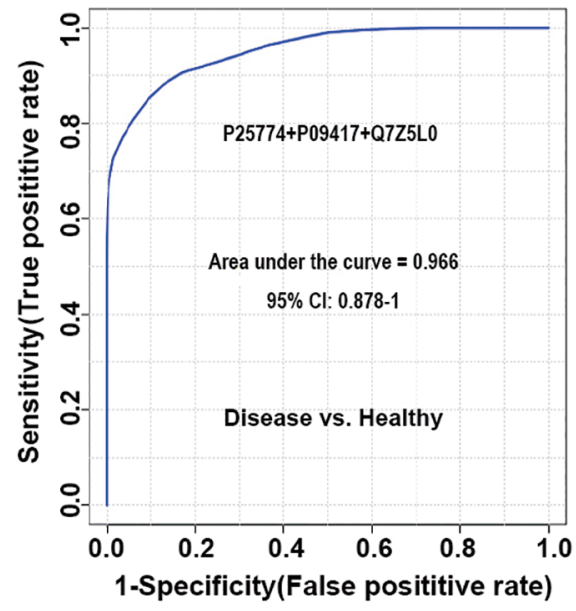

C

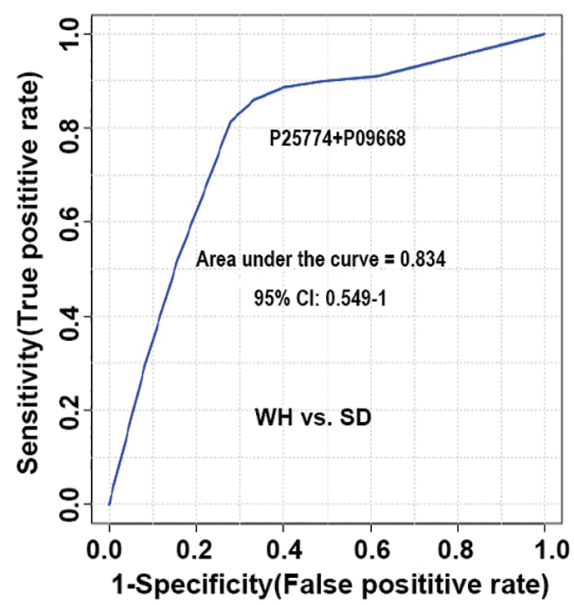

B
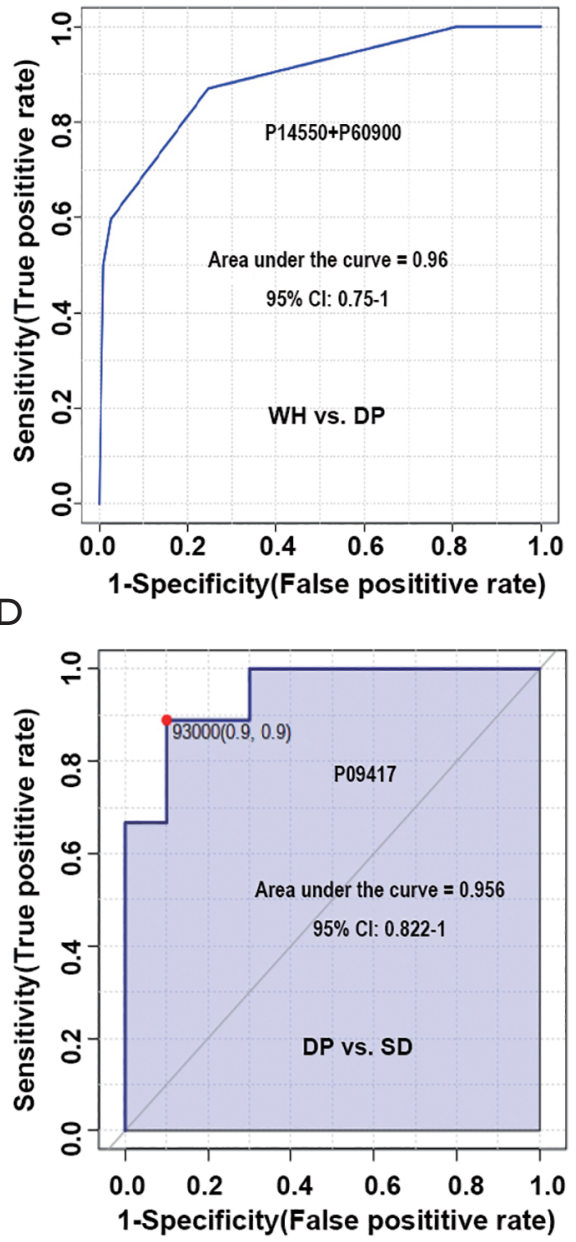

Figure 4 Diagnostic performance of biomarker panels for distinguishing abdominal type HSP and healthy controls and distinguishing three disease syndromes. The $\mathrm{x}$-axis represents the diagnostic sensitivity of the biomarker panel, and the $y$-axis represents its diagnostic specificity. (A) panel for distinguishing abdominal type HSP from healthy controls; (B) panel for distinguishing wind-heat syndrome and damppoison syndrome; (C) panel for distinguishing wind-heat syndrome and spleen-deficiency syndrome; (D) panel for distinguishing damppoison syndrome and spleen-deficiency syndrome. P25774: cathepsin S; P09417: dihydropteridine reductase; Q7Z5L0: vitelline membrane outer layer protein 1 homolog; P14550: aldo-keto reductase family 1 member A1; P60900: proteasome subunit alpha type-6; P09668: procathepsin H. CI, confidence interval; WH, wind-heat; DP, damp-poison; SD, spleen-deficiency; HSP, Henoch-Schonlein purpura.

according to the multisystem manifestations of HSP, we believe that most of the 21 diseases may have similar pathological damage. From the point of view of pathological damage, the reasoning for each protein being annotated to different disease biomarkers is shown in Table 2.

Based on the analysis in Table 2, we believe it is not surprising that abdominal type HSP shares biomarkers with most of the 21 diseases. In turn, this increases the reliability of these proteins as abdominal type HSP biomarkers. At the same time, many kidney diseases share the same biomarkers as abdominal type HSP, which suggests that in addition to presenting abdominal symptoms, abdominal type HSP patients may also have some degree of renal damage. It should be noted that the renal clinical indicators of these patients were normal. Therefore, these injuries should be minor, and urine biomarkers are very sensitive and can reflect renal changes in very early stages.

Currently, uncommon manifestations of HSP pose diagnostic difficulties. Some children may lack clinical symptoms in the early stage. Alternatively, the signs of 
Table 3 Tissue-enriched protein biomarkers and tissue involvement evidence in the pathogenesis of abdominal type HSP

\begin{tabular}{|c|c|c|c|}
\hline $\begin{array}{l}\text { Differential proteins found } \\
\text { in this study }\end{array}$ & $\begin{array}{l}\text { Number of } \\
\text { proteins }\end{array}$ & $\begin{array}{l}\text { Enriched or enhanced } \\
\text { tissue }\end{array}$ & $\begin{array}{l}\text { Evidence of tissue involvement in the pathogenesis of } \\
\text { abdominal type HSP }\end{array}$ \\
\hline $\begin{array}{l}\text { P01019; P61457; P00450; } \\
\text { P01011; P09467; P04217; } \\
\text { P52758; P17174; P09417; } \\
\text { P34896; P09210; Q9Y2S2; } \\
\text { Q5R3I4; P30046; P05062 }\end{array}$ & 15 & Liver & $\begin{array}{l}\text { Hepatobiliary involvement may happen in children with HSP (29). } \\
\text { In addition, cases of HSP with primary biliary cirrhosis (PBC) have also } \\
\text { been reported, and autoantigen-specific IgA antibody seems to play an } \\
\text { important role in their pathogenesis ( } 30)\end{array}$ \\
\hline $\begin{array}{l}\text { Q8TAB3; P09417; P21802; } \\
\text { A6NL88; O94910; Q9HC56; } \\
\text { Q9BXN2 }\end{array}$ & 7 & Brain & $\begin{array}{l}\text { Central nervous system (CNS) involvement may present as a } \\
\text { complication of HSP, which include central nervous system (CNS) } \\
\text { injury, cerebral vasculitis, cerebral hemorrhage, posterior reversible } \\
\text { encephalopathy syndrome (PRES), seizures, and peripheral nervous } \\
\text { system injury (32) }\end{array}$ \\
\hline P04406; P21695; P17174 & 3 & Skeletal muscle & HSP with muscle involvement, presenting as myositis (33) \\
\hline P09467; Q01151; Q9BXN2 & 3 & Lung & $\begin{array}{l}\text { Pulmonary involvement may be a complication of HSP and diffuse } \\
\text { alveolar hemorrhage (DAH) is the most frequent clinical presentation (34) }\end{array}$ \\
\hline P17174; P07195 & 2 & Heart muscle & $\begin{array}{l}\text { Cardiac involvement may be common in patients with HSP. IgA and C3 } \\
\text { deposited in the walls of intramyocardial vessel might account for its } \\
\text { pathogenesis (26) }\end{array}$ \\
\hline P09210 & 1 & Pancreas & Acute pancreatitis may present as a complication of HSP (35) \\
\hline P48745; Q8NHP8; P80370 & 3 & Adrenal gland & The adrenal gland may be involved in the progression of HSP (36) \\
\hline
\end{tabular}

HSP, Henoch-Schonlein purpura; GI, gastrointestinal.

uncommon manifestations may be nonspecific and masked by common complications. Therefore, the indicators of organ damage in the early stage are very useful for the diagnosis of uncommon clinical manifestations of HSP. Since urine biomarkers can reflect changes in organs in the early stage, the organ origin of the 75 differentially expressed proteins was analyzed. Because only a few proteins show strict tissue-specific expression in a single tissue or organ, we can only ascertain the tissues or organs that express the protein at a significantly elevated level. Finally, thirty-seven of the 75 proteins were found to be distributed in 13 solid tissues as tissue-enriched proteins. All 13 solid tissues can be involved in the progression of HSP. The analysis results are shown in Table 3. In the future, monitoring changes in these tissue-enriched proteins over a longer time period and in larger populations may help us identify biomarkers that can predict specific organ damage by HSP.

The IPA analysis showed that these differential proteins were correlated with the pathogenesis of abdominal type HSP (Figure 2). Macrophages, neutrophils, the immune system, and the inflammatory response were first shown to be linked with differentially expressed proteins. HSP is an immune-complex mediated vasculitis in childhood. Immune complex deposits result in necrosis of the wall of small- and medium-sized arteries with infiltration of tissue by neutrophils and deposition of nuclear fragments. This process is called leukocytoclastic vasculitis (LCV) (25). 
Neutrophils can contribute to vasculitis by increasing the release of neutrophil extracellular traps (41). Macrophages have also been found to play a decisive role in the pathogenesis of vasculitis (42). Lipid and carbohydrate metabolism were also shown to be linked with differentially expressed proteins, which might be because cellular metabolism is important in determining the immune responsiveness of lymphocytes (43). Finally, the IPA results also showed that these differentially expressed proteins can reflect multiorgan abnormalities, which was consistent with the results obtained from the tissue origin analysis of the 75 proteins.

Second, whether abdominal type HSP can be distinguished into different syndromes based on the urine proteome was investigated. PCA showed that although they could not be completely separated, there was a tendency to distinguish among the three syndromes either in DDA or DIA quantification experiments. O-PLS-DA showed that the abdominal type HSP can be classified into three syndromes based on the selected differential proteins. SD syndrome and DP syndrome were more easily separated. In the theory of traditional Chinese medicine, SD syndrome is a deficiency syndrome, while DP syndrome is an excessive syndrome. They represent opposite pathogeneses, so there will be significant differences in the proteome between the two groups.

Third, the urinary protein biomarker panel for the diagnosis and phenotyping of abdominal type HSP was constructed. As most biomarkers of HSP are not specific and originate from inflammation, immune response or vasculitis, we therefore constructed a biomarker panel to improve the diagnosis performance of HSP and facilitate its future clinical application. After using ROC analysis to evaluate the diagnostic effects of candidate biomarkers, we found that at least six differential proteins (P25774 + $\mathrm{P} 09417+\mathrm{Q} 7 \mathrm{Z} 5 \mathrm{~L} 0+\mathrm{P} 60900+\mathrm{P} 14550+\mathrm{P} 09668)$ are needed. After searching the UPBD (44), none of these six proteins in this biomarker panel had been reported as urinary disease biomarkers in previous studies.

This study is an attempt to investigate the markers of the molecular phenotype of the disease based on the urine proteome with the help of clues obtained from clinical treatment experience. The protein panel might be useful not only for the diagnosis of abdominal type HSP but also for distinguishing the three syndromes of abdominal type HSP. Furthermore, because the three disease syndromes respond differently to treatment, this biomarker panel could advantageously be used for patient stratification. Although the protein panel was constructed by the common proteins found in two urine proteome quantitative comparison experiments, it can still only be regarded as a potential biomarker, and large-scale clinical verification is needed in future experiments.

In China, to obtain better clinical outcomes, many diseases are divided into several syndromes based on traditional Chinese medicine and receive different treatments. Although use of traditional Chinese medicine theory has been controversial, all these results can be regarded as indicators when studying disease phenotypes based on modern multiomics strategies. Specifically, urine can accumulate systematic changes in the body; thus, urine proteomics has great clinical application prospects in the classification of syndromes of diseases, especially for verifying these clues. In the future, urine proteome biomarkers will be increasingly used in the classification of disease syndromes and will guide precise treatment.

\section{Acknowledgments}

Funding: This work was funded by Beijing Natural Science Foundation (7172076), National Natural Science Foundation of China (81400766) and the National Major Scientific and Technological Special Project for "Significant New Drugs Development" during the 13th Five-year Plan Period (No. 2017ZX09304029), National Key Research and Development Program of China (2018YFC0910202 and 2016YFC1306300), the Beijing Cooperative Construction Project (110651103). The funders had no role in study design, data collection and analysis, decision to publish, or preparation of the manuscript.

\section{Footnote}

Reporting Checklist: The authors have completed the MDAR reporting checklist. Available at http://dx.doi.org/10.21037/ tp-20-317

Data Sharing Statement: Available at http://dx.doi. org/10.21037/tp-20-317

Peer Review File: Available at http://dx.doi.org/10.21037/tp20-317

Conflicts of Interest: All authors have completed the ICMJE uniform disclosure form (available at http://dx.doi. org/10.21037/tp-20-317). The authors declare no conflicts of interest. 
Ethical Statement: The authors are accountable for all aspects of the work in ensuring that questions related to the accuracy or integrity of any part of the work are appropriately investigated and resolved. The study was conducted in accordance with the Declaration of Helsinki (as revised in 2013). The study was approved by the local ethics committee of Beijing Children's Hospital of Capital Medical University (No. 2016-91) and informed consent was taken from all the patients.

Open Access Statement: This is an Open Access article distributed in accordance with the Creative Commons Attribution-NonCommercial-NoDerivs 4.0 International License (CC BY-NC-ND 4.0), which permits the noncommercial replication and distribution of the article with the strict proviso that no changes or edits are made and the original work is properly cited (including links to both the formal publication through the relevant DOI and the license). See: https://creativecommons.org/licenses/by-nc-nd/4.0/.

\section{References}

1. Trnka P. Henoch-Schonlein purpura in children. J Paediatr Child Health 2013;49:995-1003.

2. Saulsbury FT. Henoch-Schonlein purpura. Curr Opin Rheumatol 2001;13:35-40.

3. Brogan P, Eleftheriou D, Dillon M. Small vessel vasculitis. Pediatr Nephrol 2010;25:1025-35.

4. Choong CK, Beasley SW. Intra-abdominal manifestations of Henoch-Schonlein purpura. J Paediatr Child Health 1998;34:405-9.

5. Saulsbury FT. Clinical update: Henoch-Schonlein purpura. Lancet 2007;369:976-8.

6. Saulsbury FT. Henoch-Schonlein purpura in children. Report of 100 patients and review of the literature. Medicine (Baltimore) 1999;78:395-409.

7. Calviño MC, Llorca J, Garcia-Porrua C, et al. HenochSchonlein purpura in children from northwestern Spain: a 20-year epidemiologic and clinical study. Medicine (Baltimore) 2001;80:279-90.

8. Trapani S, Micheli A, Grisolia F, et al. Henoch Schonlein purpura in childhood: epidemiological and clinical analysis of 150 cases over a 5-year period and review of literature. Semin Arthritis Rheum 2005;35:143-53.

9. Rosenblum ND, Winter HS. Steroid effects on the course of abdominal pain in children with Henoch-Schonlein purpura. Pediatrics 1987;79:1018-21.

10. Li B, Yang M, He GL, et al. Efficacy and Safety of
Chinese Herbs for the Prevention of the Risk of Renal Damage in Henoch-Schonlein Purpura in Children: MetaAnalysis of Randomized Controlled Trials and GRADE Evaluation. Evid Based Complement Alternat Med 2019;2019:4089184.

11. Ding Y, Zhang X, Ren X, et al. Traditional Chinese medicine versus regular therapy in Henoch-Schonlein purpura nephritis in children: study protocol for a randomized controlled trial. Trials 2019;20:538.

12. Shao C, Zhao M, Chen X, et al. Comprehensive Analysis of Individual Variation in the Urinary Proteome Revealed Significant Gender Differences. Mol Cell Proteomics 2019;18:1110-22.

13. Zhao M, Li M, Yang Y, et al. A comprehensive analysis and annotation of human normal urinary proteome. Sci Rep 2017;7:3024.

14. Ding Y, Sun X, Bi L, et al. Guidelines for diagnosis and treatment of Henoch-Schonlein Purpura in traditional Chinese medicine. Journal of Pediatrics of Traditional Chinese Medicine 2011; 7:1-4.

15. Wiśniewski JR, Zougman A, Nagaraj N, et al. Universal sample preparation method for proteome analysis. Nat Methods 2009;6:359-62.

16. Old WM, Meyer-Arendt K, Aveline-Wolf L, et al. Comparison of label-free methods for quantifying human proteins by shotgun proteomics. Mol Cell Proteomics 2005;4:1487-502.

17. Bruderer R, Bernhardt OM, Gandhi T, et al. Extending the limits of quantitative proteome profiling with dataindependent acquisition and application to acetaminophentreated three-dimensional liver microtissues. Mol Cell Proteomics 2015;14:1400-10.

18. Callister SJ, Barry RC, Adkins JN, et al. Normalization approaches for removing systematic biases associated with mass spectrometry and label-free proteomics. J Proteome Res 2006;5:277-86.

19. Zhang B, Chambers MC, Tabb DL. Proteomic parsimony through bipartite graph analysis improves accuracy and transparency. J Proteome Res 2007;6:3549-57.

20. Jennette JC, Falk RJ, Bacon PA, et al. 2012 revised International Chapel Hill Consensus Conference Nomenclature of Vasculitides. Arthritis Rheum 2013;65:1-11.

21. Pillebout E, Jamin A, Ayari H, et al. Biomarkers of IgA vasculitis nephritis in children. PLoS One 2017;12:e0188718.

22. Powell JM, Ware H, Williams G. Recurrent ureteric obstruction in association with Henoch-Schonlein purpura. Postgrad Med J 1987;63:699-701. 
23. Siomou E, Serbis A, Salakos C, et al. Masked severe stenosing ureteritis: a rare complication of HenochSchonlein purpura. Pediatr Nephrol 2008;23:821-5.

24. Guthrie RA, Guthrie DW. Pathophysiology of diabetes mellitus. Crit Care Nurs Q 2004;27:113-25.

25. Ebert EC. Gastrointestinal manifestations of HenochSchonlein Purpura. Dig Dis Sci 2008;53:2011-9.

26. Kereiakes DJ, Ports TA, Finkbeiner W. Endomyocardial biopsy in Henoch-Schönlein purpura. Am Heart J 1984;107:382-5.

27. Binstadt BA, Fleegler EW. Perforated appendicitis in a child with Henoch-Schönlein purpura. J Pediatr Surg 2005;40:E24-7.

28. Allen SJ, Sprigg A, Davidson DC. Haemorrhagic cystitis and urinary retention in Henoch-Schönlein purpura. Eur J Pediatr 1992;151:312.

29. Chao HC, Kong MS, Lin SJ. Hepatobiliary involvement of Henoch-Schönlein purpura in children. Acta Paediatr Taiwan 2000;41:63-8.

30. Gatselis NK, Stefos A, Gioti C, et al. Primary biliary cirrhosis and Henoch-Schonlein purpura: report of two cases and review of the literature. Liver Int 2007;27:280-3.

31. López-Mejías R, Castaneda S, Genre F, et al. Genetics of immunoglobulin-A vasculitis (Henoch-Schonlein purpura): An updated review. Autoimmun Rev 2018;17:301-15.

32. Pacheva IH, Ivanov IS, Stefanova K, et al. Central Nervous System Involvement in Henoch-Schonlein Purpura in Children and Adolescents. Case Rep Pediatr 2017;2017:5483543.

33. Hwang JJ, Ahn J, Kim KP, et al. Henoch-Schonlein Purpura With Muscle Involvement, Presenting as Myositis. J Clin Rheumatol 2017;23:60-2.

34. Di Pietro GM, Castellazzi ML, Mastrangelo A, et al.

Cite this article as: Jia L, Wu J, Wei J, Du L, Wang P, Zhang Y, Yu Y, Wang X, Yang Y, Gao Y. Proteomic analysis of urine reveals biomarkers for the diagnosis and phenotyping of abdominal-type Henoch-Schonlein purpura. Transl Pediatr 2021;10(3):510-524. doi: 10.21037/tp-20-317
Henoch-Schonlein Purpura in children: not only kidney but also lung. Pediatr Rheumatol Online J 2019;17:75.

35. Zhang Q, Guo Q, Gui M, et al. Henoch-Schonlein purpura with acute pancreatitis: analysis of 13 cases. BMC Pediatr 2018;18:159.

36. Landron C, Paccalin M, Chameau AM, et al. Adrenal haematoma in Henoch-Schonlein purpura. Rheumatology (Oxford) 2001;40:717.

37. Modi S, Mohan M, Jennings A. Acute Scrotal Swelling in Henoch-Schonlein Purpura: Case Report and Review of the Literature. Urol Case Rep 2016;6:9-11.

38. Nader NS, Matsumoto JMS, Lteif A. Cystic changes in the ovaries of a pre-pubertal girl with Henoch-Schönlein purpura. J Pediatr Endocrinol Metab 2010;23:517-9.

39. Iorio N, Bernstein GR, Malik Z, et al. Acute Esophageal Necrosis Presenting With Henoch-Schonlein Purpura. ACG Case Rep J 2015;3:17-9.

40. Wu TT, Sheu SJ, Chou LC, et al. Henoch-Schonlein purpura with bilateral central retinal artery occlusion. Br J Ophthalmol 2002;86:351-2.

41. Safi R, Kallas R, Bardawil T, et al. Neutrophils contribute to vasculitis by increased release of neutrophil extracellular traps in Behcet's disease. J Dermatol Sci 2018;92:143-50.

42. Shirai T, Hilhorst M, Harrison DG, et al. Macrophages in vascular inflammation--From atherosclerosis to vasculitis. Autoimmunity 2015;48:139-51.

43. Gardiner CM, Finlay DK. What Fuels Natural Killers? Metabolism and NK Cell Responses. Front Immunol 2017;8:367.

44. Shao C, Li M, Li X, et al. A tool for biomarker discovery in the urinary proteome: a manually curated human and animal urine protein biomarker database. Mol Cell Proteomics 2011;10:M111 010975. 